\title{
Hybrid Approach for Resource Scheduling in Green Clouds
}

\author{
Keffy Goyal \\ Research Fellow \\ Sri Guru Granth Sahib World University \\ Fatehgarh Sahib,INDIA
}

\author{
Supriya Kinger \\ Assistant Proffessor \\ Sri Guru Granth Sahib World University \\ FatehgarhSahib,INDIA
}

\begin{abstract}
Task scheduling is very effective issue of green Cloud computing which is used for minimization of energy consumption and for minimization of execution time. To save the power, temperature aware resource scheduler is used. It contains all features that satisfy its users and fill all task requirements.Cloud computing uses the concept of virtualization for energy efficient programs. Temperature aware resource scheduling can provide green enhancement within Cloud computing environment. In proposed algorithm the scheduler allocate the task to that machine which is far from its critical temperature as well as it consume less power. The proposed work is on a hybrid approach for both temperature and power aware resource scheduling.
\end{abstract}

Keywords- Green computing, Resource scheduling, Task scheduling, Temperature, Thermal management.

\section{INTRODUCTION}

Cloud Computing is a model for enabling ubiquitous, convenient, on-demand network access to a shared pool of configurable computing resources (e.g., networks, servers, storage, applications, and services) that can be rapidly provisioned and released with minimal management effort or service provider interaction[1]. This Cloud model promotes availability and is composed of five essential characteristics, three service models, and four deployment models. The five essential characteristics are on-demand self service, broad network access, resource pooling, rapid elasticity, and measured service. The three service models are Cloud Software as a Service (SaaS), Cloud Platform as a Service (PaaS), and Cloud Infrastructure as a Service (laaS) shown in Fig. 1.The four deployment models are Private Cloud, Community Cloud, Public Cloud, and Hybrid Cloud. These services are provided to users through data centers, virtual machines are also provided. Virtual machines make it easy to host computation and applications for large numbers of distributed users by giving each the illusion of a dedicated computer system.

Huge amount of data is stored in the data centers. So at large scale data centers consumed a lot of power [2] but It's very necessary to balance the power of the data centers. It is estimated that data centers consume $0.5 \%$ of world's total electricity usages [3]. In 2005, the total energy consumption for data centers and their cooling units were at $1.2 \%$ the total U.S energy consumption and it increases every year. Green Cloud computing is preventing the energy consumption continually grows with the growing demand of computing resources. High power consumption by data centers leads to substantial carbon dioxide emission. So there is need of Green
Cloud Computing which addresses the energy efficient resource allocation [4].

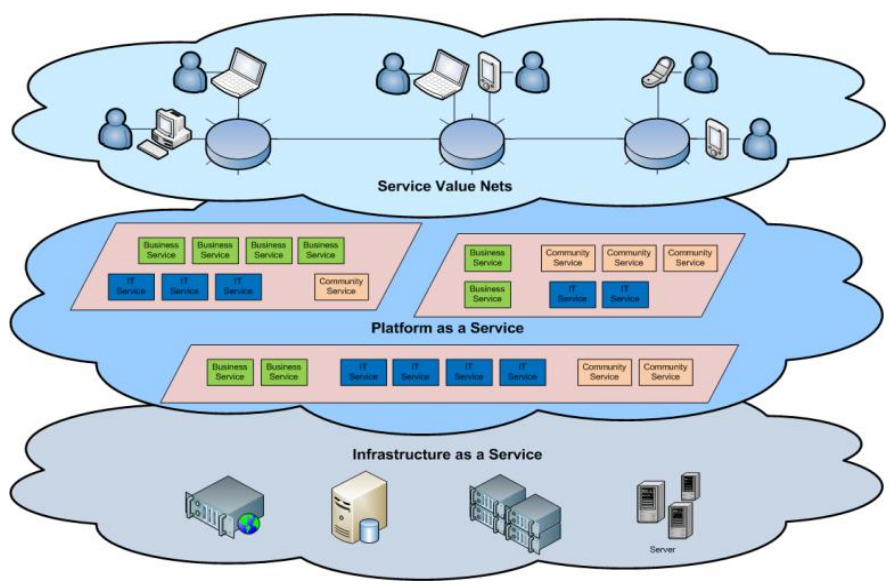

Fig. 1. Overview of cloud computing

A number of methods that can be applied to achieve high performance computing are like improvement of application's algorithm, energy efficient hardware, Dynamic voltage frequency scaling, virtualization of computing resources etc. It is also noted that the life of a computer system is directly related to its operating temperature. With the increase demand of Cloud computing the dependence on power also increases. Green computing is becoming increasingly important with the use of limited energy resources. One of the fundamental aspects of virtualization technology is resource consolidation and management using hypervisor within a cluster environment allows a number of standalone physical machines to be consolidated to a virtualized environment, thereby requiring less physical resources than ever before [5]. In this paper a green task scheduling system model is proposed for saving energy.

\subsection{Characteristics of Green Cloud Computing}

\subsubsection{Large-scalability}

The scale of green Cloud is very large .Google Cloud has owned more than one million servers and service is given to infinite users. Like Amazon, IBM, Microsoft, Yahoo, have more than hundreds of thousands servers and sprovide services. 


\subsubsection{Virtualization of green Cloud computing}

Green Cloud computing makes user to get service anywhere and anytime, through any kind of interface. One can complete all wants through net service using a computer or a mobile phone. A complete task can be quickly completed that can not be performed on a single computer.

\subsubsection{Good reliability}

Green Cloud uses data with multiplicity, it has good fault tolerant, and the computation of Cloud has very ability so it has high reliability of the service. The use of Cloud computing is more reliable than local computer.

\subsubsection{Versatility}

In Green Cloud computing many applications can run at the same time with accuracy and good Productivity.

\subsubsection{Service on demand}

Green Cloud is a big resource pool that one can buy according to the requirement and specification. Cloud is just like running water, gas, and electric that can be charged by the amount which you used.

\section{RELATED WORK}

This work reviews and discusses many approaches viz. algorithms, methods, paradigms, techniques on the issue of scheduling and allocating work load to physical machines and also concentrates on reducing energy consumption, reducing temperature and cooling cost of the data centers, optimization of workload distribution, etc.

Andrew J. Younge et al. [3] developed a new framework that provided efficient green enhancements within a scalable Cloud computing architecture. In this paper the author defined that using power-aware scheduling techniques, resource management, live migration, and a minimal virtual machine design, overall system efficiency will be greatly improved in a data centers. The results show that if the scheduler distributes the VMs with the aim to fully utilize all processing cores within each node, the power consumption is decreased dramatically.

\begin{abstract}
Anton Beloglazov et al.[6] proposed an efficient resource management policy for virtualized Cloud data centers and set threshold values for VM migration. The objective was to continuously consolidate VMs leveraging live migration and switch off idle nodes to minimize power consumption, while providing required Quality of Service. Lower and upper threshold values were set for placing the virtual machines in the host and then migration is done accordingly.
\end{abstract}

Shailesh S. Deoreet et al.[7] defined job allocation strategy to virtual machine as easy-backfilling with first come first serve basis, so amount of energy conserve.

Lathe Wang[8] proposed a method to predict a workload's thermal effect on a data center, which will be suitable for realtime scenarios and used machine learning techniques, such as artificial neural networks (ANN) as the prediction methodology and used real data taken from a data center's normal operation to conduct experiments. A thermal impact matrix to capture the special relationship between the data center's heat sources, such as the compute nodes and the environment in which theses nodes are located is introduced. The results show that machine learning techniques can predict the workload's thermal effects in a timely manner, thus making them well suited for real-time scenarios. Based on the temperature prediction techniques, developed a thermal-aware workload scheduling algorithm is for data centers, which aims to reduce power consumption and temperatures in a data center.

In this paper Lizhe Wang et al. [9], propose an analytical model, which describes data center resources with heat transfer properties and workloads with thermal features. Then two thermal aware task scheduling algorithms, TASA and TASA-B, are discussed with an aim to reduce temperatures and cooling system power consumption in a data center.

Jun Yang et al.[10] proposed an OS level technique that performs thermal aware job scheduling to reduce the number of thermal trespasses. The scheduler reduced the amount of hardware Dynamic

\section{PROPOSED WORK}

Task scheduling problems in Cloud computing are different from another scheduling problems (occurred generally) because computing services in Cloud computing are offered through a SLA between Cloud users and providers. The goal of Cloud provider is to satisfy the user requirements and to increase its profit. In this paper, a task scheduling technique is proposed which is based on system's temperature and power consumption. The technique consists of two levels. In the first level, in this an administrator whose core functions is to generate the users, different types of tasks and systems with different specifications on which the task performs. As shown in flow chart (Fig. 2).After the task is generated it is allocated to the system that matches with its requirement. Each task has some requirements like:

1. Time

2. System Requirements (CPU utilization, Memory)

3. Energy Consumption

\section{Temperature}

The paper assumes centralized scheduling scheme; i.e. a master processor unit in collecting all task, will take charge of dispatching them to the system which is most suitable for that particular task. The scheduler will generate a task/job queue. The jobs waiting in queue till the scheduler schedules it. The scheduler check the system specification like if it schedules a task on the system how much it will increase the temperature of the system. The main purpose of the scheduler is to keep the system temperature below critical temperature. It will check current temp of the system and after job execution on that system how much temp has increased at that time. If it crosses the critical temp then the scheduler goes to second system. But if that system is below the critical temperature then scheduler check the power consumption of the system. As well as it will broadcast the message to check the power consumption of all the systems. The system with less power consumption is chosen, means the task will be performed on that system. The systems generated by the administrator have its own specifications. Theses specify system feature are 
1. Critical Temperature

2. Minimum Temperature

3. Power Consumption

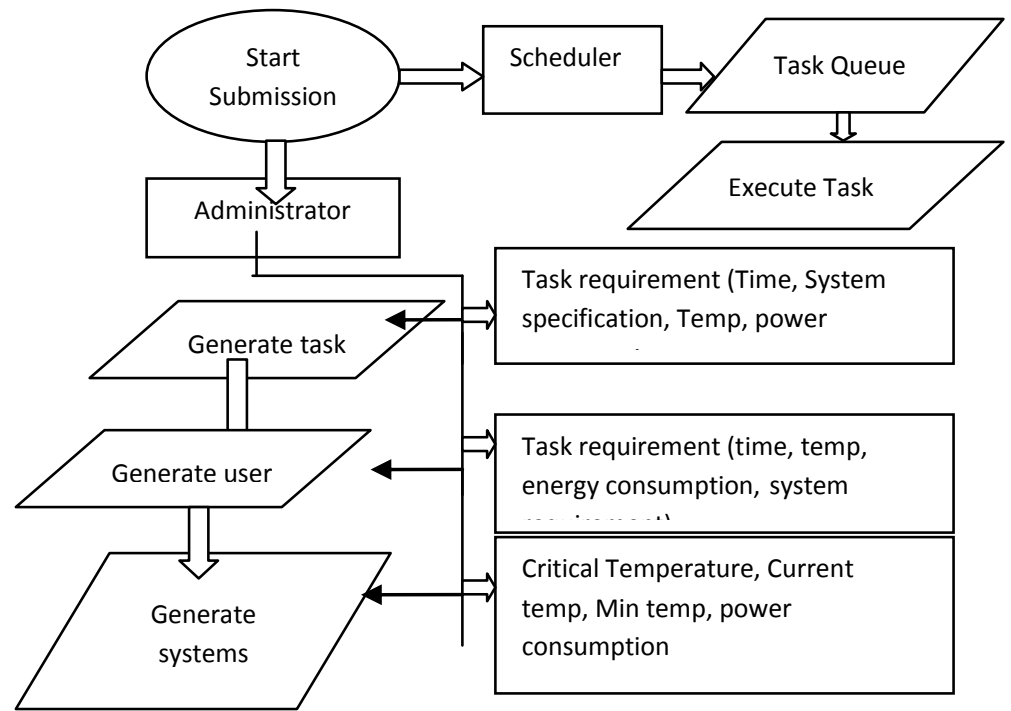

Fig.2. Thermal and Power Aware Workload Scheduling.

\subsection{Algorithm Used}

/* SS is system specification, TS task specification, System $_{i}$.TEMP is First system's temperature in the System queue, TEMP ${ }^{\max }$ Critical Temp of the System, Task ${ }_{\mathrm{i}}$ Current task ,System ${ }_{\mathrm{i}}$.power Power Consumption */

1. FOREACH Task in the Task Queue

2. If (Task Count==1) Execute Normally

3. End if

4. Else Check SS for Task $k_{\mathrm{i}} / /$ Check System Specification for First Task in the Task Queue

5. If ( $\mathrm{SS}==\mathrm{TS}) / /$ System Specification Matches With Task Specification

\section{FOREACH System in System Queue}

7. If $\left(\right.$ System $\left._{\mathrm{i}} \cdot \mathrm{TEMP}_{\mathrm{TEMP}}^{\mathrm{ma}}\right) \& \&$

$\left(\right.$ System $_{\mathrm{i}} \cdot \mathrm{TEMP}+\left(\right.$ Task $\left._{\mathrm{i}}\right)<$ TEMP $\left.^{\max }\right)$

8. Check $\left(\right.$ System $_{\mathrm{i}}$.Power $) / /$ Check the Power Consumption of the System

9. Broadcast ( )// Repeat Step 7for All Systems in System Queue

10. End if

11. If (Other System Find Power $<$ System $_{\mathrm{i}}$.Power)
12. Pass Task // Give Task to that node

13. End if

14. Else

15. Continue

16. End Else

\section{End FOREACH}

18. End Else

\section{End FOREACH}

Thermal Management achieves higher performance, while keeping the temperature low. Methods leverage the natural discrepancies in thermal behavior among different workloads, and schedule them to keep the chip temperature below a given maximum temperature. A heuristic algorithm is developed based on the observation that there is a difference in the resulting temperature when a hot and a cool job are executed in a different order. To evaluate scheduling algorithms [11], a lightweight runtime temperature monitor is developed to enable informed scheduling decisions.

\section{4 .EXPREMENTAL SETUP}

The test bed is performed on system that is having configuration 4GB RAM. In this to run the scheduler window azure portal is needed that is connected with asp.net project. Window azure is Microsoft's Cloud application platform it can be used to build a web application that runs and store its data in Microsoft data center. It allows developers to built sites using asp.net and can be deployed. System connects by changing connection string. First install SQL server $2008 \mathrm{R}_{2}$ then visual studio 2010, setup web config acc to requirement. Consider FCFS and scheduling algorithm show the comparison which is better for saving energy and temperature.

The results show in Fig. 4 demonstrated that scheduling algorithm is more energy saving as compare to FCFS.

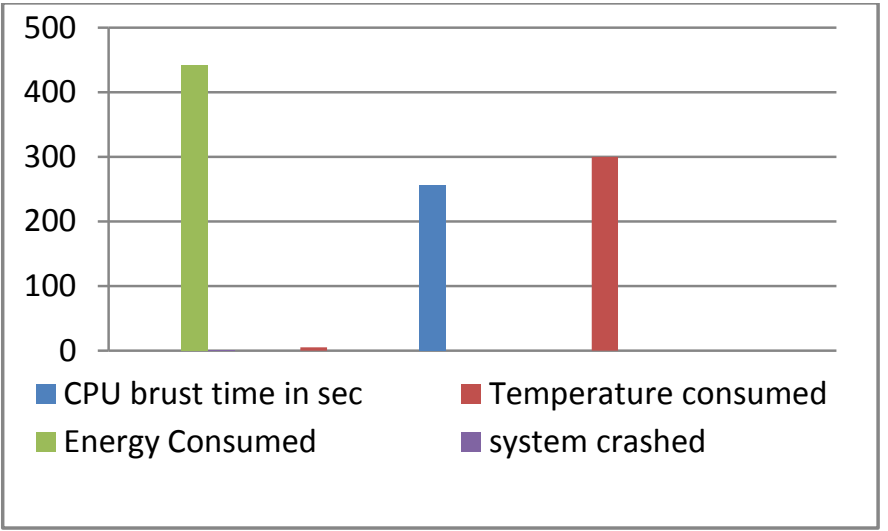

Fig. 3 Energy/Temperature consumption curve by applying FCFS policy. 


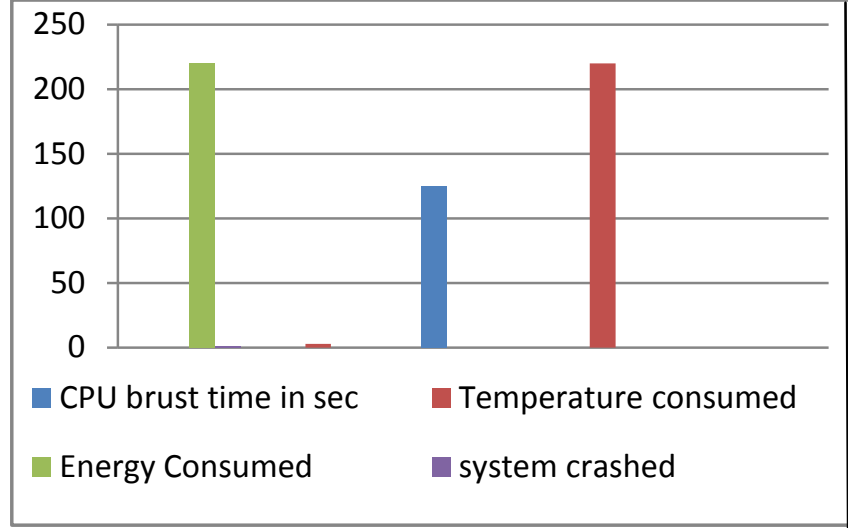

Fig. 4 Energy/Temperature consumption curve by applying our scheduling policy

The results show that energy consumption in Cloud environment is more by applying FCFS policy in Cloud data center and it is reduced by 221 watts with hybrid scheduling algorithm. As shown in Fig. 3, 5 systems burst with FCFS and by applying scheduling algorithm only 2 systems crashed this time. Energy saving is also increased with the proposed technique.

\section{THERMAL \\ AWARE TASK SCHEDULING}

Thermal aware OS task scheduling can help leverage heat and temperature. The on-chip temperature profile is hardly uniform across different functional blocks and different applications. This suggests thermal slacks for each application, which can be exploited by OS scheduler for thermal control [12]. Popular thermal-aware OS scheduling includes:

A.Dynamic Task/Thread Migration [13] it minimize the performance overhead, the scheduler loads the cores as much as possible and migrating workload when critical temperature values are observed. This Heat-and-Run approach relies on the available of cool cores when critical situation happens without any provisioning.

B. Sending Workload to Coolest Core [14] is the most intuitive way of reactive scheduling. Considering the thermal characteristics, the OS always schedule the task according to the two heuristics: Which core has the lowest temperature and which core has the coolest neighbor. This approach works well due to the correlation between workload and temperature since the coolest core usually has least workload.

C. Random Policy with Temperature Aware Adaption [14], upon arrival of each task, a probability value is calculated for each core with respect to their current workload, temperature. The scheduler assigns the task according to the probability values and generated random number.

D. The heat-balancing scheduler [11] schedules tasks according to the task queue of each core. The figure on the right illustrates the heat-balancing scheduler for two cores where a slid circle represents a hot tasks and an empty circle represents a cold task.
E. Deferring Hot Tasks [11] scheduler reactively delays the execution of certain hot task when a thermal emergency happens

\section{CONCLUSION}

Nowadays energy aware management in Cloud is becoming more important. Thermal-aware and power-aware task scheduling can offer various benefits, like reducing cooling cost and increasing reliabilities of computing resources, thus promoting an environmental conscious computing paradigm . Efficient task scheduling mechanism can meet user's requirement, and improve the resource utilization. In this paper the task scheduling mechanism is based on temperature and power consumption of the system's. The flow diagram and algorithm introduces the new methods for energy saving in cloud environment. The survey of allocating the workload, temperature of node is considered in order to reduce the $\mathrm{CO} 2$ emissions. Increase in the amount of heat may leads to destroy the machines and affects the performance also. For future work it reduces the cost of cooling and increases compute resource reliability. Aim of research is to reduce temperature of compute nodes. The idea is to distribute the workload to cool computing nodes for making thermal balancing.

\section{REFERENCES}

[1] M. Armbrust, A. Fox, R. Griffith, A.D. Joseph, R.H.Katz, A. Konwinski, G. Lee, D.A. Patterson, A. Rabkin,I.Stoica, and M. Zaharia, Above the Clouds : A Berkeley View of Cloud Computing, 2009.

[2] S. Srikantaiah, A. Kansal, and F. Zhao, "Energy aware consolidation for cloud computing," Cluster Computing, vol. 12, pp. 1-15, 2009.

[3] Andrew J. Younge et al. "Efficient Resource Management for Cloud Computing Environments" 978-1-4244-76145/10/\$26.00 @20 10 IEEE.

[4] Anton Beloglazov et al "Energy Efficient Allocation of Virtual Machines in Cloud Data Centers" 2010 10th IEEE/ACM International Conference on Cluster, Cloud and Grid Computing.

[5] S. Clearwater, Market-Based Control: A Paradigm for Distributed Resource Allocation, World Scientific.

[6] Anton Beloglazov et al "Energy Efficient Allocation of Virtual Machines in Cloud Data Centers" 2010 10th IEEE/ACM International Conference on Cluster, Cloud and Grid Computing.

[7] Shailesh S. Deore et al. "Energy-Efficient Job Scheduling and Allocation Scheme for Virtual Machines in Private Clouds" International Journal of Applied Information Systems (IJAIS) - ISSN : 2249-0868 Foundation of Computer Science FCS, New York, USA Volume 5No.1, January 2013 - www.ijais.org.

[8] Lizhe Wang et al. "Task scheduling with ANN-based temperature prediction in a data center: a simulationbased study" Engineering with Computers (2011) 27:381-391 DOI 10.1007/s00366-011-0211-4.

[9] Lizhe Wang et al. "Thermal aware workload placement with task-temperature profiles in a data center" (C) Springer Science+Business Media, LLC 2011. 
[10] J un Yang et al. "Dynamic Thermal Management through Task Scheduling" NSF grants CCF-0734339, CNS-0720595, OISE-0340752 and CCF-0641177.

[11] Zhang S, Chatha KS (2007) Approximation algorithm for the temperature-aware scheduling problem.In: ICCAD, pp 281-288

[12] J. Choi, C.-Y. Cher, H. Franke, H. Hamann, A. Weger, and P. Bose.,Thermal-aware task scheduling at the system software level, In ISLPED, 2007.

[13] M.Gomaa, M.D. Powell, and T.N. Vijaykumar, Heatand-Run: leveraging SMT and CMP to manage power density through the operating system, in ASPLOS, 2004
[14] A. K. Coskun, T. S. Rosing and K. Whisnant, Temperature Aware Task Scheduling in MPSoCs, in DATE, 2007

[15] Qinghui Tang, S.K.S. Gupta and G. Varsamopoulos, Energy-Efficient Thermal-Aware Task Scheduling for Homogeneous High-Performance Computing Data Centers: A Cyber-Physical Approach, IEEE Transactions on Parallel and Distributed Systems, vol. 19, no. 11, pp. 1458-1472, Nov 2008

[16] Y. Xie and W. Hung, Temperature-Aware Task Allocation and Scheduling for Embedded Multiprocessor System-on-Chip (MPSoC) Design, Journal of VLSI Signal
45 ,

2006 